Pacific Journal of Mathematics

A NORMAL FORM AND INTEGRATION IN FINITE TERMS
FOR A CLASS OF ELEMENTARY FUNCTIONS 


\section{A NORMAL FORM AND INTEGRATION IN FINITE TERMS FOR A CLASS OF ELEMENTARY FUNCTIONS}

\section{HeRnAN CENDRA}

Let $\mathrm{C}$ be the field of complex numbers, $E$ the usual exponential on C. So $(C, E)$ is an exponential field.

We define an exponential ring extension $\mathbf{C}\{x\}^{E}$ of $(C, E)$ and give a functional representation: $\mathbf{C}\{x\}^{E}$ is isomorphic to the smallest exponential ring extension of $(C, E)$ containing the functions $x^{s}, x$ a real and positive variable, and $s \in \mathrm{C}$.

Finally, we give a simple integration-in-finite-terms algorithm for elements of $\mathbf{C}\{x\}^{E}$.

Introduction. An "exponential ring" $(R, E)$ or " $E$-ring" is an algebraic structure with $R$ a commutative ring with 1 and $E: R \rightarrow R$ (the "exponential") a map such that

$$
\begin{aligned}
& E(a+b)=E(a) E(b) \text { for all } a, b \in R \\
& E(0)=1 .
\end{aligned}
$$

A "derivation" on $(R, E)$ is a map $D: R \rightarrow R$ such that for all $a, b \in R$ we have:

$$
\begin{aligned}
& D(a+b)=D a+D b \\
& D a b=(D a) b+a(D b) \\
& D E(a)=E(a) D a .
\end{aligned}
$$

In [5] the concept of "ring of $E$-polynomials" in the indeterminate $x$ is defined and denoted " $R[x]^{E}$ ". It is an exponential ring extension of $R$, generated as an exponential ring by the set $R \cup\{x\}$. A unique derivation $D$ can be defined on $R[x]^{E}$ such that $D r=0$ for $r \in R$, and $D x=1$. (More generally, the ring $R\left[x_{1}, \ldots, x_{n}\right]^{E}$ is defined in a similar way in [5] but we will not deal with it here.)

In $\S 1$, we define an exponential differential ring extension $R\{x\}^{E}$ of a given $(R, E)$. The ring $R[x]^{E}$ is the exponential subring of $R\{x\}^{E}$ generated by the set $R \cup\{x\}$. It should be remarked however that $R[x]^{E}$ contains an isomorphic copy of $R\{x\}^{E}$, namely $R[E(x)]^{E}$ which is the exponential subring generated by $R \cup\{E(x)\}$. 
The main purpose of this article is to give an integration-in-finite-terms algorithm for elements of $\mathbf{C}\{x\}^{E}$, with $(\mathbf{C}, E)$ the field of complex numbers with the usual exponential $E(z)=1+z / 1 !+\cdots$. Thanks are due to Professor Maxwell Rosenlicht who suggested a study of $\S 3$ (3.1) which implies in particular that if an element of $C[x]^{E}$ has an elementary integral this integral must be an element of $\mathbf{C}[x]^{E}$.

The general problem of integration in finite terms was first studied by J. Liouville [2] in the context of complex functions rather than abstract rings. There is a natural $E$-morphism $f \rightarrow \hat{f}, f \in R[x]^{E}$ onto the exponential ring $R[x]^{E}$ of functions from $R$ to $R$, generated by the identity $x$ : $R \rightarrow R$ and the constant functions $r \in R$ and such that $\hat{x}=x$ and $\hat{r}=r$. The following interesting assertions are proved in [5].

Let $R$ be an integral domain of characteristic 0 . Then

(a) The ring of constants (i.e. the ring of elements having zero derivative) of $R[x]^{E}$ is exactly $R$.

(b) The morphism " "" is an isomorphism.

In fact, an $n$-dimensional version of this is proved in [5].

In a previous version of the present paper, we gave a natural isomorphic representation

$$
\mu: \mathbf{C}\{x\}^{E} \rightarrow \mathbf{C}\{x\}^{E} .
$$

With $\mathbf{C}\{x\}^{E}$ the ring of complex valued functions of a real and positive variable $x$, generated, as an exponential ring, by constant functions $r \in \mathbf{C}$ and the functions $x^{s}=E(s \lg x)$ with $s \in \mathrm{C}$. This result was established by completely different methods to those used in [5]. In fact, it is not difficult to see that the injectivity of $\mu$ implies (a), (b) and conversely (the surjectivity of $\mu$ is a trivial fact).

Finally, we remark that the problem of integration in finite terms, even in the particular case of exponential polynomials, is undecidable in a strict sense (see [3], [4]). This is because of the impossibility of deciding equality or not between two given numbers. Of course we have such a decision method if the numbers belong to a given algebraic extension of Q.

Our algorithm reduces the integration problem to the question of deciding if certain given numerical polynomial equations are satisfied for given values of the indeterminates, and this is the best possible answer.

1. Throughout this section $(R, E)$ is an exponential ring. To simplify the exposition it will be assumed from the beginning that $R$ is an integral domain of characteristic 0 . 
We are going to define a differential exponential ring extension $R\{x\}^{E}$ of $R$.

Let $S$ be a set of indeterminates, having the same cardinal as the set $R$, and let $x \in S$ be a fixed element.

Notation. The term "monomial" will denote an expression of type $c_{1} x_{1}^{s_{1}} \cdots x_{m}^{s_{m}}, x_{i} \in S, c_{i}, s_{i} \in R$. We will write $x_{i}^{0}=1$. A "polynomial" is a sum of monomials. We will often use a notation of type " $z^{\bar{s}}$ ", with $z=x_{1} \cdots x_{m}, \bar{s}=\left(s_{1}, \ldots, s_{m}\right)$ to denote the monomial $x_{1}^{s_{1}} \cdots x_{m}^{s_{m}}=z^{\bar{s}}$. In particular we identify $z=z^{(1,1, \ldots, 1)}$.

Let $S$ be partitioned as a disjoint union:

$$
S=\{x\} \cup \bigcup_{n=1}^{\infty} S_{n}
$$

where each $S_{n}$ has the same cardinal number as the set $R$.

Define $R\{x\}_{k}^{E}, k=-1,0,1,2, \ldots$, as follows: $R\{x\}_{-1}^{E}=R . R\{x\}_{0}^{E}$ is the ring of polynomials in the indeterminate $x . R\{x\}_{n}^{E}$ is the ring of polynomials in indeterminates belonging to $\{x\} \cup S_{1} \cup \cdots \cup S_{n}$.

Let $R\{x\}^{E}$ be the ring of all polynomials in indeterminates belonging to $S$, so we have

$$
R\{x\}^{E}=\bigcup_{k=-1}^{\infty} R\{x\}_{k}^{E} .
$$

For each $n \geq 0$, choose a one-to-one surjective function $E_{n}$ from the set monomials with unit coefficient of type

$$
M=x^{s} x_{1}^{s_{1}} \cdots x_{m}^{s_{m}} \in R\{x\}_{n}^{E}-R\{x\}_{n-1}^{E}
$$

onto the set $S_{n+1}$, say: $E_{n}(M)=x_{M}$. (Note that these sets both have the same cardinal as the set $R$.) Then extend the functions $E_{n}$ to a function $E: R\{x\}^{E} \rightarrow R\{x\}^{E}$ in such a way that

$$
\begin{array}{ll}
E(a+b)=E(a) E(b) & \text { for all } a, b \in R\{x\}^{E} \\
E(0)=1 & \\
E(s M)=\left(x_{M}\right)^{s} & \text { for each monimial with unit } \\
& \text { coefficient } M, s \in R .
\end{array}
$$

So we have defined an exponential ring $R\{x\}^{E}$ which is an exponential ring extension of $R$, generated by the set $R \cup\{x\}$. 
Before we define a derivation on $R\{x\}^{E}$ we will state the following easy proposition and a definition:

1.1. Proposition. $R\{x\}_{n}^{E}$ is a free $R\{x\}_{n-k}^{E}$-module. The set of monomials in indeterminates belonging to $R\{x\}_{n}^{E}-R\{x\}_{n-k}^{E}$ is a free basis for $0 \leq k \leq n$.

In particular, we can write each $a \in R\{x\}_{n}^{E}, n \geq 0$, uniquely as follows:

$$
a=c_{1}(x) E\left(a_{1}\right)+\cdots+c_{q}(x) E\left(a_{q}\right)
$$

with $c_{i}(x) \in R\{x\}_{0}^{E}-\{0\} ; a_{i} \in R\{x\}_{n-1}^{E}$ a polynomial having constant term $0, a_{i} \neq a_{j}$ if $i \neq j$.

1.2. Definition. The expression $a=c_{1}(x) E\left(a_{1}\right)+\cdots$ is the "normal form" of the element $a$.

Using the normal form we can define by induction on $m$ a unique derivation $D$ on $R\{x\}_{n}^{E}, n \geq 1$, and then on $R\{x\}^{E}$, having the properties

$$
\begin{aligned}
& D x^{s}=s x^{s-1}, \quad s \in R, \\
& D a=0, \quad \text { if } a \in R .
\end{aligned}
$$

This completes the definition of the differential exponential ring $R\{x\}^{E}$. Now we will define a "change of variable" to get an isomorphic representation:

$$
\nu: R\{x\}^{E} \rightarrow R\left\{x_{0}\right\}^{E}
$$

with

$$
R\left\{x_{0}\right\}^{E} \subset R\{x\}^{E}
$$

as follows:

Let $x_{0}$ be the indeterminate $E(x)$ and $R\left\{x_{0}\right\}^{E}$ the exponential subring of $R\{x\}^{E}$ generated by $R \cup\left\{x_{0}\right\}$. Define " $\nu$ " as the unique exponential ring isomorphism such that $\nu \mid R=1_{R}, \nu\left(c x^{s}\right)=c x_{0}^{s}$ for all $c, s \in R$.

It is often simpler (and equivalent) to deal with $R\left\{x_{0}\right\}^{E}$ instead of $R\{x\}^{E}$. This is because the normal form for an element $a \in R\left\{x_{0}\right\}_{n}^{E}$, $n \geq 0$, has simpler coefficients $c_{i}$ and exponents $a_{i}$, namely:

$$
a=c_{1} E_{1}\left(a_{1}\right)+\cdots+c_{q} E\left(a_{q}\right)
$$


with $c_{i} \in R-\{0\} ; a_{i}$ a polynomial having constant term 0 of type

$$
a_{i} \in R\left\{x_{0}\right\}_{n-1}^{E}+\{s x\}_{s \in R}
$$

and $a_{i} \neq a_{j}$ if $i \neq j$.

REMARK. Note that $\nu$ is not a differential morphism. Moreover, we have

$$
D \nu(a)=x_{0} \nu(D) \text { for } a \in R\{x\}^{E}
$$

1.3. Proposition. The ring of constants of $R\{x\}^{E}$ is exactly $R$.

Proof. Since $R\left\{x_{0}\right\}^{E}$ is a subring of $R[x]^{E}$ it follows from (a) in the introduction that the ring of constants of $R\left\{x_{0}\right\}^{E}$ is $R$. Using the Remark, the assertion follows at once.

2. We are going to establish a formula to calculate $D f, f \in R\left\{x_{0}\right\}^{E}$, $(R, E)$ an exponential ring without zero divisors and characteristic 0 .

For a given $h \in R\left\{x_{0}\right\}^{E}$ we can effectively determine the smallest set of indeterminates, say: $\left\{x_{0}, x_{1}, \ldots, x_{m}\right\}$ such that, for some $s_{i j}, s_{k i}^{\prime} \in R$ and $s_{k} \in\{0,1\}\left(s_{k}=0\right.$ iff $\left.k \neq 0\right)$ and $c_{i} \in R$, the following equalities hold:

$$
\begin{aligned}
h & =\sum_{i} c_{i} x_{0}^{s_{0} 0} x_{1}^{s_{11}}, \ldots, x_{m}^{s_{i m}} \\
x_{k} & =E\left(x^{s_{k}} x_{0}^{s_{k 0}^{\prime}}, \ldots, x_{m}^{s_{k m}^{\prime}}\right) \quad \text { for } 0 \leq k \leq m .
\end{aligned}
$$

2.1. Definition. We will call $x_{0}, \ldots, x_{m}$ the indeterminates "associated to $h$ " and $s_{i 0}, s_{k i}^{\prime}, s_{k}$ the elements "associated to $h$ ".

The proof of the following proposition is immediate.

2.2. Proposition. The relation " $x_{i}$ associated to $x_{j}$ " is a partial order on the set of indeterminates belonging to $R\left\{x_{0}\right\}^{E}$, and $x_{0}$ is the first element. The set of indeterminates associated to $h$ is a finite set. In particular, for each $x_{i}$ associated to $h$ there exists a maximal $x_{j}$ associated to $h$ such that: $x_{i}$ associated to $x_{j}$. If $x_{i} \in R\left\{x_{0}\right\}_{n}^{E}$ and $x_{i}$ associated to $x_{j}$, $x_{i} \neq x_{j}$, then $x_{j} \in R\left\{x_{0}\right\}_{n+1}^{E}+R\left\{x_{0}\right\}_{n}^{E}$ for some $l \geq 1$. l is exactly 1 if $x_{j}$ is an immediate successor of $x_{i}$.

Notation. Let $z=x_{0}, x_{1}, \ldots, x_{m} \in R\left\{x_{0}\right\}_{n}^{E}-R\left\{x_{0}\right\}_{n-1}^{E}, n \geq 0$, with $x_{0}, \ldots, x_{m}$ the indeterminates associated to $z$. Set $z=z_{0} z_{1} \cdots z_{n}$ with $z_{i}$ the product of those indeterminates $x_{j}$ such that $x_{j} \in R\left\{x_{0}\right\}_{i}^{E}-$ $R\left\{x_{0}\right\}_{i-1}^{E}$. Let $R^{m+1}=V_{0} \oplus \cdots \oplus V_{n}$ with $V_{i}=\left\{\left(\lambda_{0}, \ldots, \lambda_{m}\right) \in R^{m+1}\right.$ : $\lambda_{j}=0$ if $x_{j}$ is not a factor of $\left.z_{i}\right\}$, and let $P_{i}: R^{m+1} \rightarrow V_{i}$ be the projection. 
By studying the expression of the derivative of an indeterminate: $x_{k}=E\left(x^{s_{k}} x_{0}^{s_{k 0}^{\prime}} \cdots x_{m}^{s_{k m}^{\prime}}\right)$ it follows that the derivative $D z^{\bar{t}}$ (for a generic $\bar{t}$ ) has a uniquely determined expression of type:

$$
D z^{\bar{i}}=\sum_{J=1}^{q} d_{J}(\bar{t}) z^{\bar{t}+\bar{\omega}_{J}} .
$$

The vectors $\bar{\omega}_{j} \in R^{m+1}$ and the $R$-linear functionals $d_{j} \neq 0, d_{j}(\bar{t})=a_{0} t_{0}$ $+\cdots a_{m} t_{m}$ can be effectively determined for a given $z$. The components of the $\bar{\omega}_{j}$ are certain sums of the $s_{k j}^{\prime}, s_{k}$, and the $a_{i}$ are certain polynomials in the $s_{k j}^{\prime}, s_{k}$.

It is clear that

$$
\begin{aligned}
D z^{i} & =\sum_{i=0}^{n}\left(\prod_{k \neq i} z^{P_{k}(\bar{i})}\right) \sum_{j=1}^{q} d_{j}\left(P_{l}(\tilde{t})\right) z^{P_{l}(\bar{t})+\bar{\omega}_{J}} \\
& =\sum_{J=1}^{q} \sum_{i=0}^{n} d_{j}\left(P_{l}(\bar{t})\right) z^{\bar{i}+\bar{\omega}_{,}} .
\end{aligned}
$$

Set $I=\{1, \ldots, q\}$ and for $0 \leq i \leq n$, set $I_{l}=\left\{j \in I: d_{j}\left(P_{l}(\bar{t})\right) \neq 0\right\}$. Observe that

$$
D z_{i}^{P_{l}(\bar{t})}=\sum_{j \in I_{l}} z_{l}^{P_{l}(t)+\bar{\omega}_{j}}
$$

We have the following (almost) obvious facts:

(i) $I_{l} \neq \varnothing$ for $i=0, \ldots, n$ (this follows from $P_{l}(t) \neq 0$ and the fact that the ring of constants, i.e. the ring of those elements having zero derivative $=R$ ). In particular, $I_{0}$ contains exactly one element, say $j \in I_{0}$ and we have: $\bar{\omega}_{j}=0$.

(ii) For $1 \leq i \leq n, j \in I_{l}$, we have $P_{l}\left(\bar{\omega}_{J}\right)=0$ for $l \geq i, P_{l-1}\left(\bar{\omega}_{J}\right) \neq 0$. (To conclude this, you only have to observe the expression of the derivative of an indeterminate.)

(iii) $I=\bigcup_{i=0}^{n} I_{l}$, disjoint union (use (ii)).

(iv) If $j \in I_{i}$ then $d_{j}(\bar{t})=d_{j}\left(P_{l}(\bar{t})\right)$ (see the above expressions of $D z^{\bar{t}}$ ).

(v) If $x_{k}$ is a maximal indeterminate in the set $\left\{x_{0}, \ldots, x_{m}\right\}$ ordered under "associated to" and if we write $\bar{\omega}_{j}=\left(\omega_{j 0}, \omega_{j 1}, \ldots, \omega_{j m}\right)$, then $\omega_{j k}=0$ for $j=1, \ldots, q$.

(vi) $x_{k}$ is associated to $z^{i}$ if and only if $x_{k}$ is associated to $D z^{\bar{i}}$. (It suffices to prove the case in which $x_{k}$ is a maximal indeterminate and this follows at once, using (v).)

The following proposition is the main result in this section. It can be easily established using the above results. 
2.2. Proposition. Let $f \in R\left\{x_{0}\right\}^{E}, f=\sum_{i=1}^{n} c_{i} z^{i i}, z=x_{0} \cdots x_{m}$ with $x_{0}, \ldots, x_{m}$ the indeterminates associated to $f$. Then

$$
D f=\sum_{i=1}^{n} \sum_{j=1}^{q} c_{i} d_{j}\left(\bar{t}_{i}\right) z^{\bar{i}_{i}+\bar{\omega}_{\jmath}}
$$

The $d_{j}, \bar{\omega}_{J}$ have the properties (i) to (vi).

3. This section deals with integration in finite terms of elements of $R\{x\}^{E}$ in the particular case $R=\mathbf{C}, E$ being an arbitrary exponential on C (for example: $E(z)=1+z / 1 !+\cdots)$.

The general case of an arbitrary $(R, E)$ with $R$ an integral domain of characteristic 0 is a consequence. In fact, only finitely generated field extensions of $\mathbf{Q}$ (the prime ring of $R$ ) are essentially involved in proofs and these are isomorphic to subfields of $\mathbf{C}$.

3.1. Proposition. Let $f \in \mathbf{C}\{x\}^{E}, f=\sum_{i=1}^{l} c_{\imath} x^{s_{i}} x_{1}^{s_{11}} \cdots x_{m}^{s_{1 m}}+c x^{-1}$ with $\left(s_{i}, s_{i 1}, \ldots, s_{i m}\right) \neq(-1,0, \ldots, 0)$ for $i=1, \ldots, l$. Then $\int f$ is elementary iff $\int\left(f-c x^{-1}\right) \in \mathbf{C}\{x\}^{E}$.

Proof. Using the "change of variable" $\nu$ we can see that the statement is equivalent to the following:

Let $f \in \mathbf{C}\left\{x_{0}\right\}^{E}, \quad f=\Sigma_{i} c_{l} x^{s_{i 0}} \cdots x_{m}^{s_{i m}}+c$ with (*) $\quad\left(s_{t 0}, \ldots, s_{i m}\right) \neq(0, \ldots, 0)$. Then $\int f$ is elementary iff $\int(f-c) \in \mathbf{C}\left\{x_{0}\right\}^{E}$.

To prove $(*)$, let $x_{0}, \ldots, x_{m}$ be the indeterminates associated to $f$; $b_{1}, \ldots, b_{q} \in \mathbf{R}$ a basis of the free $\mathbf{Z}$-module generated by the real and imaginary parts of the numbers associated to $f$, and set:

$$
\left\{U_{1}, \ldots, U_{r}\right\}=\left\{x_{\imath}^{b_{j}}, x_{k}^{\sqrt{-1} b_{l}}\right\} .
$$

The $U_{i}$ are algebraically independent over $\mathbf{C}$, and $f$ can be written

$$
f=\sum_{i} a_{i} U^{n_{i 1}} \cdots U_{r}^{n_{t r}}, \quad a_{i} \in \mathbf{C}-\{0\}, n_{i j} \in \mathbf{Z}
$$

so $f \in \mathbf{C}\left(U_{1}, \ldots, U_{r}\right)$.

$\mathbf{C}\left(U_{1}, \ldots, U_{r}\right)$ is a differential subfield of the field of quotients of C $\left\{x_{0}\right\}^{E}$. By Liouville's Theorem (see [3], [4]) we have that, for some $Q \in \mathbf{C}\left(U_{1}, \ldots, U_{r}\right), P_{k} \in \mathbf{C}\left[U_{1}, \ldots, U_{r}\right], P_{k}$ irreducible, $c_{k} \in \mathbf{C}$, we have that

$$
f=D Q+\sum_{k} c_{k} \frac{D P_{k}}{P_{k}} .
$$


Now (as in [4, Thm. 2]) we can see that if $P \in \mathbf{C}\left[U_{1}, \ldots, U_{r}\right]$ is not a monomial (recall our definition of "monomial" in $\$ 1$ ), then $P$ does not divide $D P$. In fact, let $P=\sum_{j} g_{j} U^{n_{j}} \cdots U_{p}^{n_{j p}} \in \mathbf{C}\left\{x_{0}\right\}_{n}^{E}$, with $U_{1}, \ldots, U_{r}$ $\in \mathbf{C}\left\{x_{0}\right\}_{n}^{E}-\mathbf{C}\left\{x_{0}\right\}_{n-1}^{E}, \quad U_{p+1}, \ldots, U_{r}, g_{j} \in \mathbf{C}\left\{x_{0}\right\}_{n-1}^{E}+\{0\}$; $\left(n_{j_{1}}, \ldots, n_{J_{p}}\right) \neq\left(n_{i 1}, \ldots, n_{i p}\right)$ if $i \neq j$. Then we have

$$
D P=\sum_{j} g_{j} U^{n_{j 1}} \cdots U_{p}^{n_{j p}}\left(\frac{D g_{j}}{g_{j}}+n_{j 1} \frac{D U_{1}}{U_{1}}+\cdots+n_{j p} \frac{D U_{p}}{U_{p}}\right) .
$$

If $D P$ is a multiple of $P$ then

$$
\frac{D g_{j}}{g_{j}}+n_{j 1} \frac{D u_{1}}{U}+\cdots+n_{j p} \frac{D U_{p}}{U_{p}}
$$

is independent of $j_{1}$, giving

$$
D\left(g_{l} U_{1}^{n_{11}} \cdots U_{p}^{n_{l p}} / g_{j} U_{1}^{n_{j 1}} \cdots U_{p}^{n_{j p}}\right)=0
$$

which is not true for $i \neq j$.

It follows by simple reasoning (for example, using simple partial fraction decomposition) that the $P_{k}$ are monomials, say $P_{k}=E\left(N_{k}\right)$, with $N_{k}=M_{k}+s_{k} x$, for some $M_{k} \in \mathbf{C}\left\{x_{0}\right\}^{E}, s_{k} \in \mathbf{C}$; and that $Q$ is a sum of monomials. We can conclude tht if $c=\sum_{k} s_{k}$, then

$$
\int(f-c) \in \mathbf{C}\left\{x_{0}\right\}^{E} \text {. }
$$

This finishes the proof.

3.2. Description of the algorithm. We will look at an algorithm to decide, for a given $f \in \mathbf{C}\{x\}^{E}$, if $\int f$ is elementary, and if this is the case, to find it.

As we know, we can reduce the above problem to the following: For a given $f \in \mathbf{C}\left\{x_{0}\right\}^{E}$ without constant term, decide if there is $g \in \mathbf{C}\left\{x_{0}\right\}^{E}$ such that $D g=f$, and if this is the case, find it.

Let $f=\sum_{i=1}^{M} c_{i} z^{\bar{t}_{i}}$ be given with $\bar{t}_{i} \neq 0 ; z=x_{0} x_{1} \cdots x_{m}=z_{0} z_{1} \cdots$ $z_{n}, x_{0}, \ldots, x_{m}$ the indeterminates associated to $f$ and $z_{l}$ the product of the indeterminates $x_{j} \in \mathbf{C}\left\{x_{0}\right\}_{i}^{E}-\mathbf{C}\left\{x_{0}\right\}_{i-1}^{E}$, as in $\S 2$.

If there exists $g \in \mathbf{C}\left\{x_{0}\right\}^{E}$ such that $D g=f$ we can deduce from $\S 2$ (vi) that it must be of type

$$
g=\sum_{l=1}^{N} b_{l} z^{\bar{s}_{l}}, \quad \bar{s}_{l} \neq \bar{s}_{k} \text { if } l \neq k, \quad b_{l} \in \mathbf{C}-\{0\} .
$$


So the problem finally reduces to the following:

To find $N, b_{l}, \bar{s}_{l}, l=1, \ldots, N$, such that

$$
\sum_{i=1}^{M} c_{i} z^{\bar{t}_{l}}=\sum_{l=1}^{N} \sum_{j=1}^{q} d_{j}\left(\bar{s}_{l}\right) b_{l} z^{\bar{s}_{l}+\bar{\omega}_{j}}
$$

or to establish that they do not exist.

The case $n=0$, i.e.: $f=\sum_{i=1}^{M} c_{i} z_{0}^{\bar{t}_{i}}=\sum_{i=1}^{M} c_{i} x_{0}^{\bar{t}_{i}}, t_{i} \neq 0$ is easy. In fact, we have

$$
g=\sum_{i=1}^{N}\left(\frac{c_{l}}{t_{i}}\right) \cdot x_{0}^{t_{t}} .
$$

Assume from now on that $n \geq 1$. Besides, since $P_{n}\left(\bar{\omega}_{j}\right)=0$ for $j \in I_{l}(\S 2$ (ii)), we can assume that $0 \neq P_{n}\left(\bar{t}_{i}\right)$ is independent of $i$ and that $P_{n}\left(\bar{t}_{i}\right)=$ $P_{n}\left(\bar{s}_{l}\right)$ for all $i, l$ (because we can arrange terms and decompose (**) into several equalities having this property). It follows under this assumption that if $j \in I_{n}$, then $d_{j}\left(\bar{t}_{i}\right)=d_{j}\left(P_{n}\left(\bar{t}_{i}\right)\right)=d_{j}\left(P_{n}\left(\bar{s}_{l}\right)\right)=d_{j}\left(\bar{s}_{l}\right)=d_{j}$ does not depend on $l, i$.

The general idea of the algorithm is as follows:

Choose a set of $2(m+1)$ linearly independent $\mathbf{R}$-linear functionals $\phi_{i}$ : $\mathbf{C}^{m+1} \rightarrow \mathbf{R}$ and define a linear order " $<$ " on $\mathbf{C}^{m+1}$ by means of:

$$
\begin{aligned}
& \bar{a}<\bar{b} \text { iff for some } k=1,2, \ldots, 2(m+1) \text { we have: } \\
& \phi_{k}(\bar{a})<\phi_{k}(\bar{b}) \text { and } \phi_{l}(\bar{a})=\phi_{l}(\bar{b}) \text { for } l<k .
\end{aligned}
$$

Changing the indices if necessary, we have:

$$
\bar{t}_{1}<\bar{t}_{2}<\cdots ; \quad \omega_{1}<\omega_{2}<\cdots ; \quad \bar{s}_{1}<\bar{s}_{2}<\cdots .
$$

Assume that the $\phi_{i}$ are chosen in such a way that, for some $j_{0} \in I_{n}$ we have:

$$
d_{j_{0}} \neq 0 \quad \text { and } \quad d_{j}=0 \quad \text { if } j<j_{0} .
$$

The non-zero element having lowest exponent in the matrix of elements:

$(* * *)$

$$
b_{l} d_{j}\left(\bar{s}_{l}\right) z^{\bar{s}_{l}+\bar{\omega}_{j}}
$$

is

$$
b_{1} d_{j_{0}}\left(\bar{s}_{1}\right) z^{\bar{s}_{1}+\omega 0_{j_{0}}} .
$$

From this and (**) we can deduce that: $b_{1}=c_{1} / d_{j_{0}} ; \bar{s}_{1}=\bar{t}_{1}-\bar{\omega}_{j_{0}}$.

To calculate $b_{2}, \bar{s}_{2}$, set

$$
R^{1} f=D\left(g-b_{1} z^{\bar{s}_{1}}\right)=f-D \frac{c_{1}}{d_{j_{0}}} z^{{\overline{t_{1}}}_{1} \bar{\omega}_{j_{0}}}=\sum_{i} a_{1, i} z^{\bar{U}_{1, t}}
$$


with $a_{1, i} \in \mathbf{C}-\{0\}, \bar{U}_{1,1}<\bar{U}_{1,2}<\bar{U}_{1,3}<\cdots$. Since

$$
R^{1} f=D\left(\sum_{i=2}^{N} b_{\imath} z^{\bar{s}_{t}}\right)=\sum_{i=2}^{N} \sum_{j=j_{0}}^{q} b_{i} d_{j}\left(\bar{s}_{i}\right) z^{\bar{s}_{i}+\bar{\omega}_{j}}
$$

and since terms of lowest exponents must be equal, we have

$$
b_{2}=\frac{a_{11}}{d_{j_{0}}} ; \quad \bar{s}_{2}=\bar{U}_{11}-\bar{\omega}_{J_{0}} .
$$

More generally, define

$$
R^{l} f=\sum_{i} a_{l i} z^{\bar{U}_{l l}}
$$

by induction as follows:

(1) $R^{0} f=f$

(2) If $R^{l} f \neq 0$ then

$$
R^{l+1} f=R^{l} f-D\left[\frac{a_{l 1}}{d_{j_{0}}} z^{\bar{U}_{l 1}-\bar{\omega}_{J_{0}}}\right],
$$

and, if $R^{l} f=0$ then $R^{l+1} f=0$.

We can see that, if $R^{l} f \neq 0$ then $\bar{U}_{l 1}<\bar{U}_{l+1,1}$ and $\bar{s}_{l+1}=\bar{U}_{l 1}-\bar{\omega}_{j_{0}}$; $b_{l+1}=a_{l 1} / d_{j_{0}}$. We have:

$$
R^{k} f=D\left(g-\sum_{l=1}^{k} b_{l} z^{\bar{s}_{l}}\right)=f-\sum_{l, j} b_{l} d_{j}\left(\bar{s}_{l}\right) z^{\bar{s}_{l}+\bar{\omega}_{J}} .
$$

A useful picture of the situation is the following: $R^{k} f$ is the difference of $f$ minus the elements belonging to the $k$ first columns of the matrix of elements $(* * *)$. Observe that only the elements belonging to the same ascending diagonal may have equal exponents. In particular, since $\bar{s}_{N}+$ $\bar{\omega}_{q}>\bar{s}_{l}+\bar{\omega}_{j}$ if $j<q$ or $l<N$, we have that $b_{N} d_{q}\left(\bar{s}_{N}\right) z^{\bar{s}_{N}+\bar{\omega}_{1}}$ could be cancelled only with some term $c_{l} z^{\bar{t}_{i}}$ of $f$ and not with some $b_{l} d_{j}\left(\bar{s}_{l}\right) z^{\bar{s}_{l}+\bar{\omega}_{j}}$.

It is clear that if we repeatedly apply the algorithm, we will finally reach a step, say $N$, such that one and only one of the following assertions holds:
(A) $R^{N-1} f \neq 0, R^{N} f=0$
(B) $R^{l} f \neq 0$ for all $l \geq N$.

If (A) holds, then $g=\sum_{l=1}^{N} b_{l} z^{\bar{z}_{l}}$ is a solution of $D g=f$. If (B) holds, the problem $D g=f$ has no solution.

Now we will see how to calculate a bound $\tilde{N}$ for $N$. $\tilde{N}$ has the property that if we reach the step $\tilde{N}$ and $R^{\tilde{N}} f \neq 0$, then we have: $R^{l} f \neq 0$ for $l \geq \tilde{N}$, which implies that we are in case (B). So, if there is a solution it must be found at a certain step $N \leq \tilde{N}$. 
We will introduce the following notation:

$$
j_{2}(\bar{a})=\max \left\{j: d_{j}(\bar{a}) \neq 0\right\}
$$

so in the expression

$$
D g=\sum_{i, j} d_{j}\left(\bar{s}_{l}\right) z^{\bar{s}_{l}+\bar{\omega}_{j}}
$$

the sum is extended to $1 \leq j \leq j_{2}\left(\bar{s}_{l}\right)$. Besides, we define

$$
J=J_{1} \cup\left(I-I_{n}\right), \quad \text { with } J_{1}=\left\{j \in I_{n}: d_{j} \neq 0\right\}
$$

(observe that $J_{1} \neq 0$ ).

There are two cases to be considered regarding the position of the vectors $\bar{\omega}_{j}$ in the $\mathbf{C}^{m+1}$ space. The idea is as follows: Given $f$ we have a $z$ determined as explained before and, hence, the $\bar{\omega}_{j}$ are also determined. Then we must check if we are in Case 1 or in Case 2, which will depend on the position of the vectors $\bar{\omega}_{j}$ in $\mathbf{C}^{m+1} \cong \mathbf{R}^{2(m+1)}$ space. Then we have to choose the functionals $\phi_{1}, \ldots, \phi_{2(m+1)}$ to fill the condition assumed for the case we are in. All this is a matter of elementary geometry in $\mathbf{C}^{m+1}$. To easily understand the description of the algorithm, it will be useful to take into account a few remarks.

Let $\mathbf{C}^{m+1}=v_{0} \oplus \cdots \oplus v_{n}, P_{i}: \mathbf{C}^{m+1} \rightarrow V_{i}$ be the projection as in $\S 2$. A given $\mathbf{R}$-linear function $\phi: \mathbf{R}^{2(m+1)} \rightarrow \mathbf{R}$ can always be written: $\phi(\bar{x})=$ $(\bar{x}, \bar{a})$, “ $($,$) " meaning standard inner product in \mathbf{R}^{2(m+1)}$. For given vectors $\bar{b}_{1}, \bar{b}_{2}, \ldots$ we can choose $\bar{a}$ and the indices " $j$ " of the $\bar{b}$, in such a way that $\phi\left(\bar{b}_{1}\right)<\phi\left(\bar{b}_{2}\right)<\cdots$.

The following observations will be especially useful in Case 2. Assume that $\phi_{1}(x)=(x,-v)$, and that $\phi_{1}\left(\bar{\omega}_{1}\right) \leq \phi_{1}\left(\bar{\omega}_{2}\right) \leq \cdots$. We can find an arbitrarily small vector $\bar{\varepsilon}$ such that

$$
\text { If } \phi_{2}(x)=(\bar{x},-\bar{v}+\bar{\varepsilon}) \text { then } \phi_{2}\left(\bar{\omega}_{1}\right)<\phi_{2}\left(\bar{\omega}_{2}\right) \cdots \text {. }
$$

Moreover, if we know that $\bar{v} \in V_{n-1}$ and $\phi_{1}\left(\bar{\omega}_{j}\right) \leq 0, j \in J$, we can deduce (using $\S 2$ (ii)) that $\bar{\varepsilon}$ can be chosen in such a manner that: $\bar{\varepsilon} \in V_{0} \oplus \cdots V_{n-2}$. If, in addition, we fix $j_{1} \in I_{n-1}$, we can choose $\bar{\varepsilon}$ in such a manner that $j \geq j_{1}$ implies $j \in I_{n-1}$. (If $n>2$ it is enough to choose $\bar{\varepsilon}=\bar{\varepsilon}^{\prime}+\bar{\varepsilon}^{\prime \prime}$ with $\bar{\varepsilon}^{\prime}$ having the same direction and sense as $P_{n-2}\left(\bar{\omega}_{J_{1}}\right)$ and $\bar{\varepsilon}^{\prime \prime} \in V_{0} \oplus \cdots V_{n-3}$ small. If $n=2, I_{n-2}=\{j\}=I_{0}$.)

Case 1. In this case we assume that there are at least two indices $j, l \in I_{n}$ such that $P_{n-1}\left(\bar{\omega}_{j}\right), P_{n-1}\left(\bar{\omega}_{l}\right) \neq 0$, do not have the same direction and sense. This implies that we can choose an R-linear function such that 
(changing indices if necessary),

(1) $\phi_{1}\left(\bar{t}_{1}\right)<\phi_{1}\left(\bar{t}_{2}\right)<\cdots ; \phi_{1}\left(\bar{\omega}_{1}\right)<\phi_{1}\left(\bar{\omega}_{2}\right)<\cdots$

(2) For some $j_{0}, j_{1} \in J_{1}$ we have:

(i) $j_{0}<j_{1}$

(ii) $j<j_{0}$ or $j>j_{1}$ implies $j \in I_{n}$ and $d_{J}=0$.

Now we choose $\phi_{2}, \ldots, \phi_{2(m+1)}$ in such a manner that $\left\{\phi_{1}, \ldots, \phi_{2(m+1)}\right\}$ is a linearly independent set and we define the linear order $<$ as we have already explained.

By looking at the definition of $R^{l} f$ it can be shown that $\bar{U}_{l, 1}=\bar{t}_{i_{l}}+\bar{\omega}_{l}^{\prime}$ with $\bar{t}_{i_{l}} \in\left\{\bar{t}_{1}, \ldots, \bar{t}_{n}\right\}$ and $\bar{\omega}_{l}^{\prime}$ a sum having $L_{l}^{\prime}$ terms of type $\bar{\omega}_{j}-\bar{\omega}_{J_{0}}$, $j \in J, j>j_{0}$. We can therefore deduce that, for a given $L$, there exists a number $l=l(L)$ such that: If $l>l(L)$ and $R^{l-1} f \neq 0$ then $L_{l}^{\prime}>L$. (Because, if for each given $l_{0}$, there is an $l>l_{0}$ such that $R^{l-1} f \neq 0$ and $L_{l}^{\prime}<L$, then the number of terms in the sequence $\bar{U}_{1,1}<\bar{U}_{2,1}<\cdots$ remains bounded, a contradiction, since it is an increasing sequence.)

It follows from the previous facts that

$$
\phi_{1}\left(\bar{U}_{l 1}\right) \geq \min _{i} \phi_{1}\left(t_{i}\right)+L_{l}^{\prime} \phi_{1}\left(\bar{\omega}_{j_{0}+1}-\bar{\omega}_{J_{0}}\right)
$$

and then we do reach a step, say $\tilde{N}$, such that either (A) holds, or

$$
\phi_{1}\left(\bar{U}_{\tilde{N}, 1}-\bar{\omega}_{j_{0}}+\bar{\omega}_{j_{1}}\right)>\max _{i} \phi_{1}\left(\bar{t}_{i}\right) \text {. }
$$

The last possibility implies that the nonero term:

$$
b_{\tilde{N}+1} d_{J}\left(\bar{s}_{\tilde{N}}\right) z^{\bar{s}_{\tilde{N}+1}+\bar{\omega}_{11}}
$$

of highest exponent in the expression of $R^{\tilde{N}} f$ cannot be cancelled neither with a term of type $b_{l} d_{j}\left(\bar{s}_{l}\right) z^{\bar{s}_{l}+\bar{\omega}_{J}} \neq 0$, because $\bar{s}_{l}+\bar{\omega}_{j}<\bar{s}_{\tilde{N}}+\bar{\omega}_{j_{1}}$ nor with a term $c_{i} z^{\bar{t}_{i}}$. We can conclude that $R^{N} f \neq 0$. Moreover, we have

$$
\phi_{1}\left(U_{\tilde{N}+1,1}-\bar{\omega}_{j_{0}}+\bar{\omega}_{j_{1}}\right) \geq \phi_{1}\left(\bar{U}_{\tilde{N} 1}-\bar{\omega}_{J_{0}}+\bar{\omega}_{J_{1}}\right)>\max _{i} \phi_{1}\left(\bar{t}_{l}\right) .
$$

As before, we can deduce from this that $R^{\tilde{N}+1} f \neq 0$. It is clear that we could repeat the process, and then, prove that

$$
\phi_{l}\left(\bar{U}_{l 1}-\bar{\omega}_{j_{0}}+\bar{\omega}_{j_{1}}\right)>\max _{i} \phi_{1}\left(\bar{t}_{l}\right) \text { for } l>N
$$

and finally, we can conclude that $R^{l} f \neq 0$ for every $l$, so (B) holds. So $\tilde{N}$ is the bound we were looking for.

Case 2. For some $\bar{v} \in V_{n-1},|\bar{v}|=1$, holds: $P_{n-l}\left(\bar{\omega}_{j}\right)=\lambda_{j} \bar{v}$, we have $\lambda_{j} \geq 0$ for $j \in J$ and $\lambda_{j}>0$ iff $j \in J_{1}$. Thus this case is opposite to the previous one. 
The general idea and notation are as in Case 1 ; to find $\tilde{N}$ is a bit more delicate. We must first choose carefully $\phi_{1}, \phi_{2}$ and then get (by any procedure) a linearly independent set: $\left\{\phi_{1}, \phi_{2}, \ldots, \phi_{2}(m+1)\right\}$.

First of all, we choose $\phi_{1}(\bar{x})=(\bar{x},-\bar{v})$. Now we choose $\phi_{2}(\bar{x})=$ $(\bar{x},-\bar{v}+\bar{\varepsilon})$ such that (changing the indices if necessary) the following assertions hold:

$$
\phi_{2}\left(\bar{\omega}_{1}\right)<\phi_{2}\left(\bar{\omega}_{2}\right)<\cdots ; \quad \phi_{1}\left(\bar{\omega}_{1}\right) \leq \phi_{1}\left(\bar{\omega}_{1}\right) \leq \cdots ;
$$
$\phi_{2}\left(\bar{t}_{1}\right)<\phi_{2}\left(\bar{t}_{2}\right)<\cdots$. As a consequence we have $j \in$ $J_{1}, i \notin I_{n}$ implies $j<i$. (Observe that $\phi_{1}\left(\bar{\omega}_{j}\right)<0$ and $\phi_{1}\left(\bar{\omega}_{i}\right)=0$.)

$j_{0}=\min ,\{j \in J\} \quad$ (definition of $j_{0}$ ). Then we have $j_{0} \in J_{1}$ (observe that $\phi_{1}\left(\bar{\omega}_{j}\right)=-\lambda_{j}<0, j \in J_{1}$ ). $j_{0}^{\prime}=$ $\max _{j}\left\{j \in J: \quad \phi_{1}\left(\bar{\omega}_{j}\right)=\phi_{1}\left(\bar{\omega}_{j_{0}}\right)\right\} \quad$ (definition of $j_{0}^{\prime}$ ). Then $j_{0}^{\prime} \in J_{1} \cdot j_{0}^{\prime \prime}=\min _{j}\left\{j \in J: \quad \phi_{1}\left(\bar{\omega}_{j_{0}}\right)<\phi_{1}\left(\bar{\omega}_{J}\right)\right\}$ (definition of $\left.j_{0}^{\prime \prime}\right) \cdot j_{1}=\max _{j}\left\{j \in I: \quad d_{j}(\bar{v}) \neq 0\right\}$ (definition of $j_{1}$ ). We have: $j_{1} \in I_{n-1}$ (in fact, $d_{j}(\bar{v})$ $=0$ for $j \notin I_{n-1}$ since $\left.\bar{v} \in V_{n-1}\right)$.

From the above definitions and remarks we can deduce: $j_{0} \leq j_{0}^{\prime}<j_{1} ; j \leq j_{0}^{\prime}$ implies $j \in I_{n}$. Besides, we can (1") choose $\phi_{2}$ in such a way that $j \in J$ and $j \geq j_{1}$ implies $j \in I_{n-1}$.

It can be shown, by using the definition of $R^{\prime} f$, that: $\bar{U}_{l, 1}=\bar{t}_{l l}+\bar{\omega}_{l}^{\prime}+$ $\bar{\omega}_{l}^{\prime \prime}$, with $\bar{\omega}_{l}^{\prime}$ a sum of a number, say $L_{l}^{\prime}$ of terms $\bar{\omega}_{J}-\bar{\omega}_{j_{0}}, j \in J, j>j_{0}^{\prime}$; $\bar{\omega}_{l}^{\prime \prime}$ a sum of a certain number, say $L_{l}^{\prime \prime}$ of terms of type $\bar{\omega}_{J}-\bar{\omega}_{J_{0}}, j \in J$, $j_{0}<j \leq j_{0}^{\prime}$, and $\bar{t}_{i l} \in\left\{\bar{t}_{1}, \ldots, \bar{t}_{n}\right\}$.

We can see that the following facts hold:

(2) For a given $L>0$, there is an $l(L)$ such that if $l>l(L)$ and $R^{l-1} f \neq 0$ then $L_{l}^{\prime}+L_{l}^{\prime \prime}>L$ (proof as in Case 1$)$.

(3) If $R^{l-1} f \neq 0$, then

$$
\begin{aligned}
& \max _{i} \phi_{1}\left(\bar{t}_{i}\right)+L_{l}^{\prime} \phi_{1}\left(\bar{\omega}_{q}-\bar{\omega}_{J_{0}}\right) \\
& \geq \phi_{1}\left(\bar{U}_{l, 1}\right) \geq \min _{i} \phi_{1}\left(\bar{t}_{l}\right)+L_{l}^{\prime} \phi_{1}\left(\bar{\omega}_{J_{0}^{\prime \prime}}-\bar{\omega}_{J_{0}}\right) .
\end{aligned}
$$

(To prove this, observe that $\phi_{1}\left(\bar{\omega}_{l}^{\prime \prime}\right)=0$.)

(4) If $R^{I-1} f \neq 0$, then

$$
\begin{aligned}
\max _{i} \phi_{2}\left(\bar{t}_{l}\right)+ & \left|\left(L_{l}^{\prime}+L_{l}^{\prime \prime}\right)\right|\left|\phi_{2}\left(\bar{\omega}_{q}-\bar{\omega}_{j_{0}}\right)\right| \\
& \geq \phi_{2}\left(\bar{U}_{l 1}\right) \geq \min \phi_{2}\left(\bar{t}_{i}\right)+\left|\left(L_{l}^{\prime}+L_{l}^{\prime \prime}\right)\right| \phi_{2}\left(\bar{\omega}_{j_{0}+1}-\bar{\omega}_{j_{0}}\right)
\end{aligned}
$$


(5) If $R^{l-1} f \neq 0$, then:

$$
\left|d_{j_{1}}\left(\bar{U}_{l, 1}-\bar{\omega}_{j_{0}}\right)\right| \geq-\max _{i}\left|d_{j_{1}}\left(\bar{t}_{i}-\bar{\omega}_{j_{0}}\right)\right|+L_{l}^{\prime}\left|\lambda_{j_{0}^{\prime \prime}}-\lambda_{j_{0}}\right| \cdot\left|f_{j_{1}}(\bar{v})\right| .
$$

(Note that for $j \in J$ holds):

$$
d_{J_{1}}\left(P_{n-1}\left(\bar{\omega}_{j}-\bar{\omega}_{j_{0}}\right)\right)=d_{j_{1}}\left(\left(\lambda_{j}-\lambda_{j_{0}}\right)(\bar{v})\right)=\left(\lambda_{j}-\lambda_{j_{0}}\right) \cdot d_{j_{1}}(\bar{v}),
$$

and also recall from $\S 2$ (iv) that

$$
d_{j_{1}}\left(P_{n-1}\left(\bar{\omega}_{j}-\bar{\omega}_{j_{0}}\right)\right)=d_{j_{1}}\left(\bar{\omega}_{j}-\bar{\omega}_{j_{0}}\right) .
$$

Now we will use these facts to establish our algorithm in Case 2. First of all, assume $j_{0}=j_{0}^{\prime}$. Then $\bar{\omega}_{l}^{\prime}+\bar{\omega}_{l}^{\prime \prime}=\bar{\omega}_{l}^{\prime}, L_{l}^{\prime \prime}+L_{l}^{\prime}=L_{l}^{\prime}$. Using (2), (3) we can show that we reach a step, say $\tilde{N}$, such that either (A) holds or $\phi_{1}\left(\bar{U}_{\tilde{N}, 1}-\bar{\omega}_{j_{0}}+\bar{\omega}_{j_{1}}\right)>\phi$, with

$$
\begin{aligned}
\phi= & \max _{i} \phi_{1}\left(\bar{t}_{i}\right)+\left(\max _{i} \frac{d_{j_{1}}\left(\bar{t}_{t}-\bar{\omega}_{j_{0}}\right)}{\left(\lambda_{j_{0}^{\prime \prime}}-\lambda_{j_{0}}\right) d_{j_{1}}(\bar{v})}\right) \phi_{1}\left(\bar{\omega}_{q}-\bar{\omega}_{j_{0}}\right) \\
& +\phi_{1}\left(\bar{\omega}_{j_{1}}-\bar{\omega}_{j_{0}}\right) .
\end{aligned}
$$

Now we will see that if this last possibility holds, then (B) holds.

First we will see that

$$
0 \neq R^{\tilde{N}+1} f=f-\sum_{l, j} b_{l} d_{j}\left(\bar{s}_{l}\right) Z^{\bar{s}_{l}+\bar{\omega}_{J}}, \quad 1 \leq l \leq \tilde{N}+1 ; j_{0} \leq j \leq j_{2}\left(\bar{s}_{l}\right) .
$$

Using (3) and the relation $\phi\left(\bar{U}_{\bar{N}_{1}}-\bar{\omega}_{j_{0}}+\bar{\omega}_{j_{1}}\right)>\phi$ we can deduce that $d_{j_{1}}\left(\bar{U}_{N, 1}-\bar{\omega}_{j_{0}}\right)=d_{j_{1}}\left(\bar{s}_{\tilde{N}+1}\right) \neq 0$. So, if we set $j_{2}=j_{2}\left(\bar{s}_{\tilde{N}+1}\right)$, we have $j_{2} \geq j_{1}$ and then $\phi_{1}\left(\bar{s}_{\tilde{N}+1}+\bar{\omega}_{j_{2}}\right) \geq \phi>\max _{i} \phi_{i}\left(\bar{t}_{i}\right)$. Therefore: $\bar{s}_{\tilde{N}+1}+\bar{\omega}_{j_{2}}>\bar{t}_{i}$.

Besides, if $l<\tilde{N}+1, j>j_{2}, j \in J, d_{j}\left(\bar{s}_{l}\right) \neq 0$ and $\bar{s}_{l}+\bar{\omega}_{j}=\bar{s}_{\tilde{N}+1}$ $+\bar{\omega}_{j_{2}}$, then (by $\left.\left(1^{\prime \prime}\right)\right), j \in I_{n-1}$ and therefore

$$
d_{j}\left(\bar{s}_{\tilde{N}+1}\right)=d_{j}\left(P_{n-1}\left(\bar{s}_{l}+\bar{\omega}_{j}-\bar{\omega}_{j_{2}}\right)\right)=d_{j}\left(P_{n,-1}\left(\bar{s}_{l}\right)\right)=d_{j}\left(\bar{s}_{l}\right) \neq 0,
$$

a contradiction (see the definition of $j_{2}(\bar{x})$ ). We have proved that the term $b_{\tilde{N}+1} d_{j_{2}}\left(\bar{s}_{\tilde{N}+1}\right) Z^{s_{\tilde{N}+1}+\bar{\omega}_{j 2}}$ of $R^{\tilde{N}+1} f$ cannot be cancelled, so $R^{\tilde{N}+1} f \neq 0$. Besides since $\bar{U}_{\tilde{N}+1,1}>\bar{U}_{\tilde{N}, 1}$ we have

$$
\phi_{1}\left(\bar{U}_{\tilde{N}+1,1}-\bar{\omega}_{j_{0}}+\bar{\omega}_{j_{1}}\right) \geq \phi_{1}\left(U_{\tilde{N}, 1}-\bar{\omega}_{j_{0}}+\bar{\omega}_{j_{1}}\right)>\phi,
$$

and therefore we can iterate and finally we can conclude that (B) holds. We have established the case $j_{0}=j_{0}^{\prime}$. 
Now assume that $j_{0}^{\prime}>j_{0}$. We will see the following assertion: a step, say $\tilde{N}$, is reached such that one (and only one) of the following conditions holds:

(a) $R^{\tilde{N}-1} f \neq 0$ and $R^{\tilde{N}} f=0$ (condition (A))

(b) $R^{\tilde{N}} f \neq 0$ and $\phi_{1}\left(\bar{U}_{\tilde{N}, 1}-\bar{\omega}_{j_{0}}+\bar{\omega}_{j_{1}}\right)>\phi$

( $\phi$ as in the case $j_{0}^{\prime}=j_{0}$ ).

(c) $R^{\tilde{N}} f \neq 0$ and for some $N>\tilde{N}$ we have

$$
\phi_{1}\left(\bar{U}_{\tilde{N}, 1}\right)=\phi_{1}\left(\bar{U}_{\tilde{N}+1,1}\right)=\cdots=\phi_{1}\left(\bar{U}_{N, 1}\right)
$$

and

$$
\phi_{2}\left(\bar{U}_{N, 1}-\bar{\omega}_{j_{0}}+\bar{\omega}_{j_{0}^{\prime}}\right)>\psi_{\tilde{N}},
$$

with $\psi_{\tilde{N}}=\max \left\{\phi_{2}\left(\bar{U}_{\tilde{N}, l}\right): l \in B_{N}\right\}$ with $B_{\tilde{N}}=\left\{l: \phi_{1}\left(\bar{U}_{\tilde{N}, l}\right)=\phi_{1}\left(U_{\tilde{N}, 1}\right)\right\}$.

To prove this, assume that there is not a number $\tilde{N}$ such that (a) or (b) holds, and therefore, that $R^{l} f$ remains nonero and $\phi_{1}\left(\bar{U}_{L, 1}\right)$ remains bounded. Then (by (3)), $L_{l}^{\prime}$ remains bounded. On the other hand, we have (since $\left.\phi_{1}\left(\bar{\omega}^{\prime \prime}\right)=0\right), \phi_{1}\left(\bar{U}_{l, 1}\right)=\phi_{1}\left(\bar{t}_{i l}\right)+\phi_{1}\left(\bar{\omega}_{l}^{\prime}\right)$. We can deduce from these facts that $\phi_{1}\left(\bar{U}_{l, 1}\right)$ takes only a finite number of values and then that there is a number $\tilde{N}$ such that the sequence $\phi_{1}\left(\bar{U}_{0,1}\right) \leq \phi_{1}\left(\bar{U}_{1,1}\right) \leq \cdots \leq$ $\phi_{1}\left(\bar{U}_{l, 1}\right) \leq \cdots$ remains constant for $l \geq \tilde{N}$, and besides (by (2) and (4)) we reach a step, say $N$, such that $N>\tilde{N}$ and $\phi_{2}\left(\bar{U}_{N, 1}-\bar{\omega}_{j_{0}}+\bar{\omega}_{J_{0}^{\prime}}\right)>\psi_{\tilde{N}}$. We have proved the assertion.

If (b) holds, we can prove as in case $j_{0}=j_{0}^{\prime}$ that (B) holds.

Now assume that $(\mathrm{C})$ holds. We therefore have

$$
R^{N+1} f=R^{\tilde{N}} f-\sum_{l, j} b_{l} d_{j}\left(\bar{s}_{l}\right) Z^{\bar{s}_{l}+\bar{\omega}_{J}}
$$

$$
\tilde{N}+1 \leq l \leq N+1, j_{0} \leq j \leq j_{2}\left(\bar{s}_{l}\right) .
$$

If $\bar{s}_{N+1}+\bar{\omega}_{j_{0}^{\prime}}=\bar{U}_{\tilde{N}, l}$, we can deduce: $\phi_{1}\left(\bar{U}_{\tilde{N}, l}\right)=\phi_{1}\left(\bar{U}_{N, 1}\right)=\phi_{1}\left(\bar{U}_{\tilde{N}, 1}\right)$, so $l \in B_{\tilde{N}}$. It follows that $\phi_{2}\left(\bar{s}_{N+1}+\bar{\omega}_{j_{0}^{\prime}}\right)>\psi_{\tilde{N}} \geq \phi_{2}\left(U_{\bar{N}_{\tilde{N}} l}\right)$, a contradiction. Now assume that, for some $l, j(j \in J)$ such that $\tilde{N}+1 \leq l<N+1$, $j_{0}^{\prime}<j \leq j_{2}\left(\bar{s}_{l}\right)$, we have that $\bar{s}_{l}+\bar{\omega}_{j}=\bar{s}_{N+1}+\bar{\omega}_{j_{0}^{\prime}}$. Then

$$
\phi_{1}\left(\bar{U}_{l-1,1}-\bar{\omega}_{j_{0}}+\bar{\omega}_{j}\right)=\phi_{1}\left(\bar{U}_{N, 1}-\bar{\omega}_{j_{0}}+\bar{\omega}_{j^{\prime}}\right)=\phi_{1}\left(\bar{U}_{N, 1}\right) .
$$

Now, since $\phi_{1}\left(\bar{\omega}_{j}-\bar{\omega}_{j_{0}}\right)>0$ we can deduce that $\phi_{1}\left(\bar{U}_{l-1,1}-\bar{\omega}_{j_{0}}+\bar{\omega}_{j}\right)>$ $\phi_{1}\left(\bar{U}_{l-1,1}\right)=\phi_{1}\left(\bar{U}_{N, 1}\right)$, a contradiction. So we have proved that $R^{N+1} f \neq 0$.

Now for some $l \geq 1$, say $l=l_{0}$, we have that $\bar{s}_{N+1}+\bar{\omega}_{j_{0}^{\prime}}=\bar{U}_{N+1, l_{0}}$, so we can deduce that $\phi_{1}\left(\bar{U}_{N+1,1}\right) \leq \phi_{1}\left(\bar{U}_{N, 1}\right)$. We can deduce from this (since $\left.\bar{U}_{N, 1}<\bar{U}_{N+1,1}\right)$ that: $\phi_{1}\left(\bar{U}_{N+1,1}\right)=\phi_{1}\left(\bar{U}_{N, 1}\right)$ and $\phi_{2}\left(\bar{U}_{N, 1}\right) \leq \phi_{2}\left(\bar{U}_{N+1,1}\right)$ so we can conclude that $\phi_{2}\left(\bar{U}_{N+1,1}-\bar{\omega}_{J_{0}}+\bar{\omega}_{j_{0}^{\prime}}\right)>\psi_{\tilde{N}}$. It is clear that we can iterate and prove that $R^{\tilde{N}+l} f \neq 0$ for every integer $l$, so (B) holds. 


\section{REFERENCES}

[1] I. Kaplansky, An Introduction to Differential Algebra, (Hermann, Paris, 1957).

[2] J. Liouville, Mémoire sur les transcendantes elliptiques de première et de seconde espèce, considerés comme fonctiones de leur amplitude, J. Ecole Polytech., 14 (1833), 57-83.

[3] R. Risch, The problem of integration in finite terms, Trans. Amer. Math. Soc., 139 (1969), 167-189.

[4] M. Rosenlicht, Differential extension fields of exponential type, Pacific J. Math., 57, No. 1 (1975), 289-300.

[5] L. van den Dries, Exponential rings, exponential polynomials, and exponential functions, Pacific J. Math., 113, No. 1 (1984), pg. 51.

Received July 21, 1982 and in revised form April, 4, 1985.

UNIVERSITY OF CALIFORNIA

BERKELEY, CA 94720 


\section{PACIFIC JOURNAL OF MATHEMATICS EDITORS}

V. S. VARADARAJAN (Managing Editor)
University of California
Los Angeles, CA 90024
HEBERT CLEMENS
University of Utah
Salt Lake City, UT 84112
CHARLES R. DEPRIMA
California Institute of Technology
Pasadena, CA 91125

\section{R. FINN}

Stanford University

Stanford, CA 94305

HeRmanN FlaschKa

University of Arizona

Tucson, AZ 85721

RAMESH A. GANGOLli

University of Washington

Seattle, WA 98195

ROBION KIRBY

University of California

Berkeley, CA 94720

\section{C. MoOre}

University of California

Berkeley, CA 94720

H. SAMELSON

Stanford University

Stanford, CA 94305

HAROLD STARK

University of California, San Diego

La Jolla, CA 92093

ASSOCIATE EDITORS
R. ARENS
E. F. BECKENBACH
B. H. NeUmanN
F. WOLF
K. Yoshida (1906-1982)

\section{SUPPORTING INSTITUTIONS}

UNIVERSITY OF ARIZONA

UNIVERSITY OF BRITISH COLUMBIA

CALIFORNIA INSTITUTE OF TECHNOLOGY

UNIVERSITY OF CALIFORNIA

MONTANA STATE UNIVERSITY

UNIVERSITY OF NEVADA, RENO

NEW MEXICO STATE UNIVERSITY

OREGON STATE UNIVERSITY
UNIVERSITY OF OREGON

UNIVERSITY OF SOUTHERN CALIFORNIA

STANFORD UNIVERSITY

UNIVERSITY OF HAWAII

UNIVERSITY OF TOKYO

UNIVERSITY OF UTAH

WASHINGTON STATE UNIVERSITY

UNIVERSITY OF WASHINGTON 


\section{Pacific Journal of Mathematics}

\section{Vol. 121, No. $1 \quad$ November, 1986}

Om P. Agrawal, Douglas Napier Clark and Ronald George Douglas,

Invariant subspaces in the polydisk $\ldots \ldots \ldots \ldots \ldots \ldots \ldots \ldots \ldots \ldots$

Christoph Bandt and Gebreselassie Baraki, Metrically invariant measures

on locally homogeneous spaces and hyperspaces $\ldots \ldots \ldots \ldots \ldots \ldots \ldots$

Marcy Mason Barge, Horseshoe maps and inverse limits ..............29

Russell Gene Bilyeu, Robert Richard Kallman and Paul Weldon Lewis,

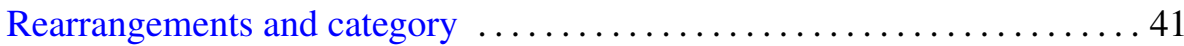

Jean Bourgain, A problem of Douglas and Rudin on factorization . . .......47

Hernan Cendra, A normal form and integration in finite terms for a class of

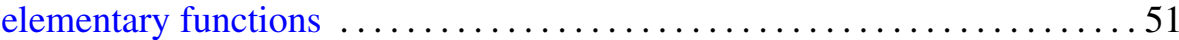

Ky Fan, The angular derivative of an operator-valued analytic function . . . . 67

Gerhard Gierz, On the Dunford-Pettis property of function modules of

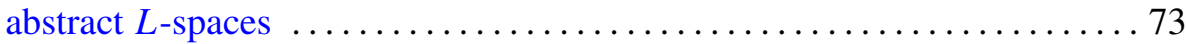

Gabriel Katz, On polynomial generators in the algebra of complex functions

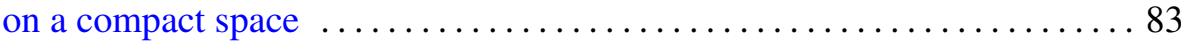

Ridgley Lange, Duality and asymptotic spectral decompositions $\ldots \ldots \ldots . .93$

Anthony To-Ming Lau and Peter F. Mah, Quasinormal structures for certain spaces of operators on a Hilbert space ................... 109

R. Daniel Mauldin, Correction: "The set of continuous nowhere differentiable functions"

Alan Harvey Mekler and Saharon Shelah, $\omega$-elongations and Crawley's problem

Alan Harvey Mekler and Saharon Shelah, The solution to Crawley's problem

Richard Rochberg, Deformation of uniform algebras on Riemann surfaces

Joseph Roitberg, On weak epimorphisms in homotopy theory

Jesús M. Ruiz, A remark on fields with the dense orbits property

Henry Wente, Counterexample to a conjecture of H. Hopf

David G. Wright, Rigid sets in $E^{n}$ 